\title{
Effects of components of semen extenders on the binding of stallion spermatozoa to bovine or equine zonae pellucidae
}

\author{
Marco A Coutinho da Silva, George E Seidel Jr ${ }^{1}$, Edward L Squires ${ }^{2}$, James K Graham ${ }^{1}$ and \\ Elaine M Carnevale ${ }^{1}$ \\ Department of Veterinary Clinical Sciences, The Ohio State University, Columbus, Ohio 43210, USA, ${ }^{1}$ Animal \\ Reproduction and Biotechnology Laboratory, Colorado State University, Fort Collins, Colorado 80523, USA and \\ ${ }^{2}$ Gluck Equine Research Center, University of Kentucky, Lexington, Kentucky 40546, USA
}

Correspondence should be addressed to M A Coutinho da Silva; Email: marco.dasilva@cvm.osu.edu

\begin{abstract}
The effects of semen extender components on the ability of stallion sperm to bind to the zona pellucida (ZP) and the suitability of using bovine ZP for a ZP-binding assay for stallion sperm were investigated in a series of experiments. In Experiment I, binding of stallion sperm to both bovine and equine ZP was significantly increased when a skim milk-based extender (EZM) was used. In Experiment II, a threefold increase in sperm binding to ZP was observed when sperm were diluted in EZM compared with diluents, which contained no milk (TALP, LAC, and EmCare). In Experiment III, centrifuging the sperm through Percoll did not increase sperm binding to the ZP but did remove any positive effect of EZM on sperm-ZP binding. In Experiment IV, exposure of either sperm or ZP to EZM before co-incubation did not increase sperm binding to ZP. In Experiment V, sperm diluted in TALP containing skim milk, EZM, or INRA96 bound more efficiently to the ZP than sperm diluted in TALP without milk proteins. In Experiment VI, sodium caseinate, native phosphocaseinate, and caseinoglycopeptide increased sperm binding to the ZP. In conclusion, diluents containing milk or milk proteins markedly enhanced the number of sperm bound to both equine and bovine ZP.

Reproduction (2012) $\mathbf{1 4 3} 577-585$
\end{abstract}

\section{Introduction}

Binding of sperm to the zona pellucida (ZP) plays a crucial role in the process of fertilization. In the oviduct, sperm penetrate the mass of cumulus cells surrounding the oocyte and bind to the ZP, the last physical barrier that sperm must overcome before fertilization. Inability of sperm to penetrate the ZP inevitably results in infertility (Green 1997). The initial binding of sperm to the ZP is loose but specific receptor-ligand pairing on the surfaces of the sperm head and the ZP leads to tight linkage of the gametes (Yanagimachi 1994). However, despite intense investigation, the molecular mechanism of sperm binding to the ZP has yet to be determined (He et al. 2003, Dean 2004, Hoodbhoy \& Dean 2004).

ZP-binding assays have been used to estimate the fertilizing capacity of sperm in several species. Human sperm binding to homologous ZP highly correlates with male fertility and outcomes of IVF (Liu et al. 1989, Oehninger et al. 1989, Gamzu et al. 1994). In similar experiments, binding ability of sperm to the ZP correlated with fertility of bulls (Fazeli et al. 1993), boars (Ivanova \& Mollova 1993, Braundmeier et al. 2004), and stallions (Fazeli et al. 1995, Pantke et al. 1995, Meyers et al. 1996). However, in the horse, use of
ZP-binding assays has been limited because of the scarcity of equine oocytes. Therefore, development of a ZP-binding assay for the horse using heterologous ZP would be a useful tool to study determining elements involved in fertilization.

During natural mating or artificial insemination of mares, semen is deposited within the uterine body, and sperm are transported to the site of fertilization in the oviduct. For some assisted reproductive technologies in the horse, such as gamete intrafallopian transfer (GIFT), sperm are deposited directly into the oviduct, and components of semen extenders may be present during gamete interaction. In the mare, GIFT has been performed in our laboratory using fresh, cooled, and frozen stallion semen (Coutinho da Silva et al. 2002, 2004). Pregnancy rates obtained with cooled and frozen semen were significantly lower than those with fresh semen $(25,8$, and $82 \%$ respectively). During GIFT with fresh, cooled, or frozen semen, sperm were used raw (no extender) or diluted with skim milk-based extender and lactose-EDTA extender (LAC; Cochran et al. 1984) respectively. We hypothesize that components of semen extenders could be responsible for the reduced pregnancy rates. 
Table 1 Mean \pm S.E.M. numbers of sperm bound to bovine or equine ZP in Experiment I.

\begin{tabular}{lcc}
\hline Treatment & ZP $(n)$ & Mean \pm s.E.M. \\
\hline BOV-TALP & 40 & $29 \pm 5^{*}$ \\
EQ-TALP & 37 & $38 \pm 7^{*}$ \\
BOV-EZM & 40 & $149 \pm 9^{+}$ \\
EQ-EZM & 40 & $151 \pm 17^{+}$
\end{tabular}

${ }^{*}{ }^{+}$Values with different superscripts differ $(P<0.05)$.

Therefore, the objectives of this study were to i) determine the suitability of bovine ZP for a ZP-binding assay with stallion sperm and ii) investigate the effects of components commonly used in semen extenders on sperm binding to $\mathrm{ZP}$ in vitro.

\section{Results}

In Experiment I, stallion sperm bound firmly to both bovine and equine ZP (Table 1). In addition, considerably more sperm bound to bovine and equine ZP when sperm were diluted with EZM compared with TALP (Fig. 1).

In Experiment II, similar numbers of sperm bound to ZP when LAC, TALP, and Emcare Holding Solution (EHS) were used (Table 2). However, sperm binding to ZP was significantly higher when sperm were diluted with EZM.

In Experiment III, centrifugation through a Percoll gradient did not affect the number of sperm bound to ZP when sperm were diluted with TALP (Table 3). Higher number of sperm was bound to the ZP when sperm in EZM were centrifuged and suspended in EZM compared with TALP. However, this effect was negated when sperm in EZM were centrifuged through Percoll and suspended in $\operatorname{TALP}(\mathrm{EZM}+\mathrm{P})$.

In Experiments IV-A and IV-B, sperm binding to the ZP was significantly higher when sperm were suspended in EZM (Table 4). In Experiment IV-A, centrifugation of sperm diluted with EZM with subsequent suspension in TALP eliminated the positive effect on sperm binding to the ZP. In Experiment IV-B, preincubation of ZP in a solution of $10 \%$ EZM in TALP (ZP-EZM) did not increase the number of sperm bound to the ZP. Therefore, the number of sperm bound to the ZP was only increased when sperm and ZP are co-incubated in the presence of $10 \%$ EZM.

In Experiment V, glucose had no significant effect on the number of sperm bound to ZP (Table 5). However, extenders containing milk or milk protein (INRA, EZM, and TSM) caused a significant increase in the number of sperm bound to ZP compared with TALP.

In Experiment $\mathrm{VI}$, milk proteins enhanced sperm binding to ZP (Table 6). The number of sperm bound to CG1 was similar to TALP alone. Furthermore, sodium caseinate (SC) and native phosphocaseinate (NP) were more effective in increasing sperm binding than caseinoglycopeptide (CG).
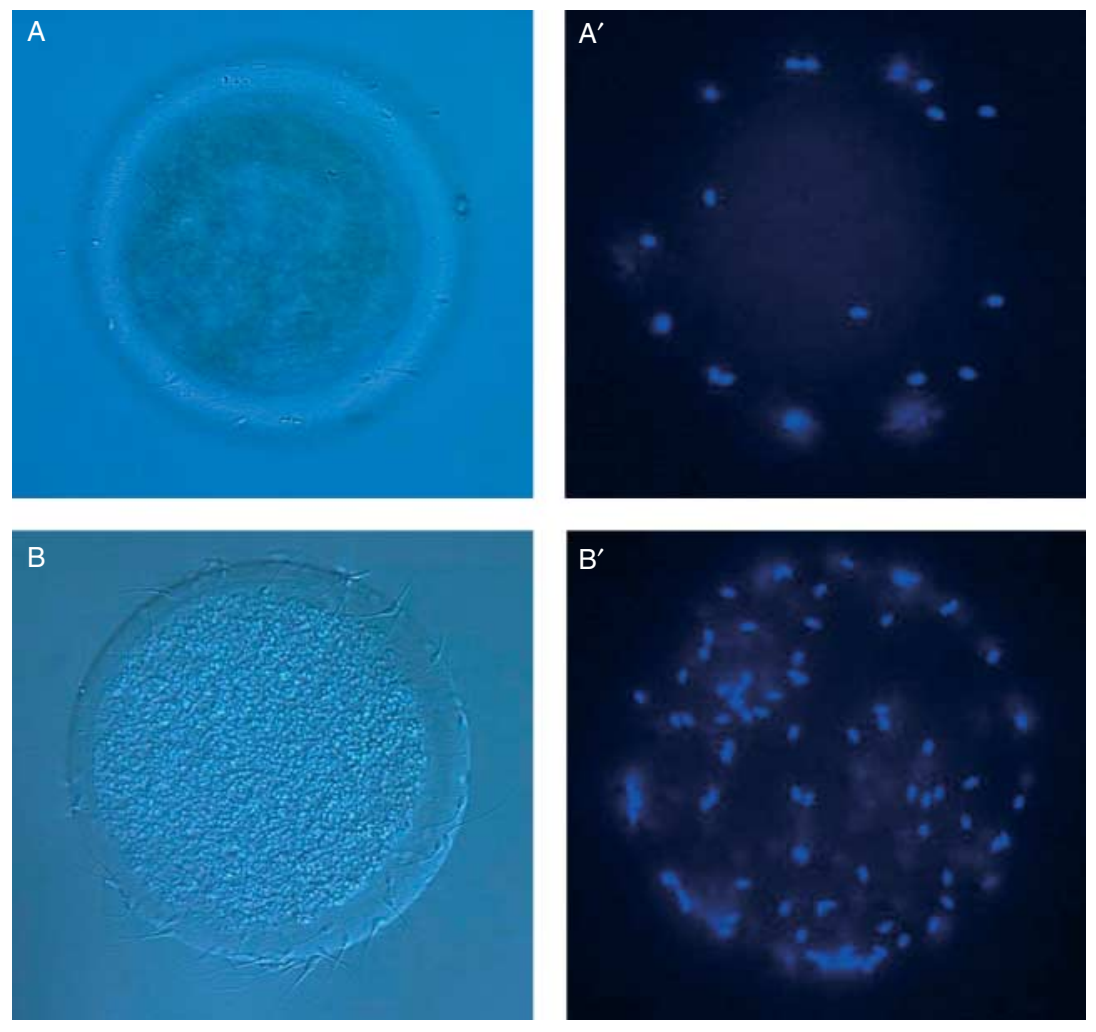

Figure 1 Light microscopy (A and $B$ ) and fluorescence microscopy $\left(A^{\prime}\right.$ and $\left.B^{\prime}\right)$ of bovine $\mathrm{ZP}$ co-incubated with equine sperm for $2 \mathrm{~h}$. More sperm bound to ZP when sperm were diluted with EZM ( $\mathrm{B}$ and $\mathrm{B}^{\prime}$ ) compared with TALP ( $\mathrm{A}$ and $\left.\mathrm{A}^{\prime}\right)$. Sperm were stained with Hoechst 33342 and observed at $400 \times$ magnification. 
Table 2 Mean \pm s.E.M. number of sperm bound per bovine ZP for various semen extenders in Experiment II.

\begin{tabular}{lcc}
\hline Treatment & ZP $(n)$ & Mean \pm s.E.M. \\
\hline LAC & 53 & $29 \pm 7^{*}$ \\
TALP & 56 & $32 \pm 8^{*}$ \\
EHS & 59 & $38 \pm 6^{*}$ \\
EZM & 60 & $96 \pm 10^{+}$ \\
\hline
\end{tabular}

${ }^{*}+$ Values with different superscripts differ $(P<0.05)$.

There was a significant $(P<0.05)$ ejaculate effect for Experiments I, III, IV-A, V, and VI.

\section{Discussion}

With the exception of some cross-species hybrids, the process of fertilization with normal embryo development is highly species specific. However, early events of gamete recognition and binding appear to be less restrictive in some species. Equine sperm are able to bind firmly to ZP of bovine oocytes, undergo the acrosome reaction, penetrate the $\mathrm{ZP}$, and fuse with the ooplasm (Sinowatz et al. 2003). In contrast, Mugnier et al. (2009) demonstrated that porcine ZP is more selective than equine $\mathrm{ZP}$ with fewer stallion sperm binding to porcine vs equine ZP. Based on these results, it appears that bovine ZP supports heterologous sperm binding and would be a better candidate for use in a heterologous ZP-binding assay. Therefore, in Experiment $\mathrm{I}$, we have investigated the use of bovine $\mathrm{ZP}$ for a ZP-binding assay using stallion sperm. We observed similar treatment differences in a number of stallion sperm bound to bovine and equine ZP. The number of sperm bound to ZP was approximately fourfold higher for both bovine and equine ZP when sperm were diluted with skim milk-based extender (EZM) than TALP, demonstrating the suitability of bovine ZP for subsequent ZP-binding experiments. Moreover, TALP was used in all subsequent experiments as a control extender because TALP is a chemically defined medium and optically clear.

In this study, salt-stored bovine and equine ZP were used. Yanagimachi et al. (1979) demonstrated that salt storage of oocytes from several species, including humans, preserved various physical and chemical characteristics of the ZP. In addition, Fazeli et al. (1995) demonstrated a relationship between sperm binding to ZP of salt-stored oocytes and stallion fertility. Therefore, storage of oocytes in a salt solution has not affected binding of sperm to ZP.

A significant ejaculate effect was observed in Experiments I, III, IV-A, V, and VI, and this effect could be potentially attributed to variation in seminal plasma between ejaculates. However, immediately after collection, seminal plasma was removed by centrifugation, and its concentration was estimated to be $<1 \%$ in the final sperm sample. In addition, sperm samples were further diluted tenfold during co-incubation with
ZP. The ejaculate effect observed in the above experiments was likely due to variations in assay conditions and/or characteristics of $Z \mathrm{P}$, as ejaculates were processed on different days, and multiple batches of ZP were used.

Experiment II investigated the effects of semen extenders previously used for equine GIFT (Coutinho da Silva et al. 2002, 2004) on sperm binding to ZP. During GIFT, sperm are deposited directly into the oviduct; therefore, seminal extenders could affect gamete interactions. The number of sperm bound to the ZP was threefold higher when sperm were diluted with EZM; LAC and EHS did not affect sperm binding to ZP. This is in contrast with the results obtained after GIFT using sperm diluted with EZM and cooled for $24 \mathrm{~h}$. We obtained similar embryo development rates when cooled sperm diluted in EZM (noncentrifuged) or EHS (centrifuged) were used for GIFT $(1 / 6,17 \%$ vs $3 / 10,30 \%$; Coutinho da Silva et al. 2004). However, overall pregnancy rates obtained with GIFT using cooled semen $(4 / 16,25 \%)$ were lower than when fresh semen was used $(9 / 11,82 \%)$, suggesting that the reduction in pregnancy rates observed was likely due to differences in the semen used (cooled vs fresh) and not to differences in extenders (EZM vs EHS). In the present experiment, only fresh semen was used, and potentially, changes in sperm during the cooling process could have negated the positive effect of EZM on sperm binding to ZP. Further studies are necessary to determine the effects of cooling on sperm binding to ZP in the presence of milk proteins.

Sperm preparation for assisted reproductive technologies usually involves selection of highly motile sperm and removal of seminal plasma, debris, and contaminants. In the horse, Percoll density gradients have been used to select populations of highly motile sperm (Del Campo et al. 1990, Carnevale et al. 1999, 2000, Alvarenga \& Leao 2002, Coutinho da Silva et al. 2002, 2004, Galli et al. 2002, Lazzari et al. 2002, Nie et al. 2003). Arns \& Shepherd (1994) demonstrated that a higher number of sperm bound to equine ZP after centrifugation through a Percoll gradient. The authors concluded that increased sperm binding to ZP was caused by initiation of capacitation during centrifugation through the Percoll gradient. These results are in disagreement with our findings in Experiment III; we observed that centrifugation through Percoll had no effect on sperm binding to ZP. In addition, when sperm diluted in EZM were centrifuged through Percoll and

Table 3 Effect of centrifugation through Percoll on sperm binding to ZP.

\begin{tabular}{lcc}
\hline Treatment & ZP $(n)$ & Mean \pm s.E.M. \\
\hline TALP & 38 & $20 \pm 6^{*}$ \\
TALP+P & 35 & $25 \pm 4^{*}$ \\
EZM & 39 & $140 \pm 23^{+}$ \\
EZM+P & 38 & $32 \pm 17^{*}$ \\
\hline
\end{tabular}

${ }^{*}{ }^{+}$Values with different superscripts differ $(P<0.05)$. Mean \pm s.E.M. number of sperm bound per bovine ZP in Experiment III. 
Table 4 Effect of the presence of EZM during sperm-ZP interaction.

\begin{tabular}{lcc}
\hline Treatment & ZP $(n)$ & Mean \pm s.E.M. \\
\hline Exp. IV-A & & \\
TALP & 38 & $20 \pm 6^{*}$ \\
EZM & 39 & $140 \pm 23^{+}$ \\
SP-EZM & 37 & $29 \pm 9^{*}$ \\
Exp. IV-B & & \\
TALP & 40 & $29 \pm 5^{*}$ \\
EZM & 40 & $149 \pm 9^{+}$ \\
ZP-EZM & 40 & $28 \pm 5^{*}$
\end{tabular}

${ }^{*},+$ Values within experiments with different superscripts differ $(P<0.05)$. Mean \pm S.E.M. numbers of sperm bound per bovine ZP in Experiment IV.

${ }^{\text {a }}$ Sperm preincubated in EZM and resuspended in TALP. ${ }^{\mathrm{b}}$ ZP preincubated in EZM and washed in TALP before insemination with sperm diluted in TALP.

resuspended in TALP $(E Z M+P)$, the positive effect of EZM on sperm binding was negated. The increase in sperm binding observed by Arns \& Shepherd (1994) could have been caused by the use of a different medium (Hams F-10) containing 3\% (w/v) BSA, which is tenfold higher than the concentration of BSA used in this study. It has been postulated that BSA serves as a cholesterol acceptor, stimulating efflux of cholesterol from the sperm plasma membrane, resulting in changes in plasma membrane fluidity and leading to capacitation (Go \& Wolf 1985, Hoshi et al. 1990, Gamzu et al. 1997, Osheroff et al. 1999, Visconti et al. 1999a, 1999b, 1999c). Therefore, the increase in sperm binding observed by Arns \& Shepherd (1994) could be attributed to initiation of sperm capacitation by BSA and not by Percoll.

In Experiments I, II, and III, sperm binding to ZP increased when sperm and ZP were incubated in TALP containing $10 \%$ EZM (i.e. $5 \mu \mathrm{l}$ sperm diluted in EZM were added to $45 \mu$ I TALP containing the ZP). When sperm diluted with EZM were centrifuged through Percoll and suspended in TALP (Experiment III), the increase in sperm binding was not observed. Therefore, Experiment IV was designed to determine whether 10\% EZM in medium during sperm and ZP interaction was necessary to enhance sperm binding to ZP. In Experiment $I V-A$, consistent with the previous experiments, sperm binding to ZP was enhanced when sperm and ZP were co-incubated in the presence of $10 \%$ EZM. However, the positive effect of EZM on sperm binding was negated when sperm pre-exposed to EZM were suspended in TALP, suggesting that components of EZM were directly affecting sperm-ZP interactions or ZP. In Experiment $I V-B$, no increase in sperm binding was observed when $Z P$ were pre-exposed to EZM and washed in TALP before assay. Therefore, we concluded that EZM needed to be present during sperm and ZP co-incubation to cause an increase in sperm binding and components of EZM directly affected the interaction of sperm and ZP.

Formulation of EZM was based on Kenney et al.'s (1975) study and consisted of dried skim milk (24 g/l), a high concentration of glucose $(272 \mathrm{mM})$, and antibiotics. Sperm binding to ZP increased in the presence of $10 \%$ EZM. Therefore, in Experiment $\mathrm{V}$, TALP was modified to contain a similar concentration of skim milk and increasing concentrations of glucose. In addition, INRA96, an extender that contains only one milk protein, was used. Glucose did not increase sperm binding to ZP. In contrast, a threefold increase in sperm binding to ZP was observed with skim milk-based extenders and INRA. A similar increase in sperm binding to the ZP was observed with EZM and INRA. INRA contains one milk protein, NP; therefore, effects of NP on sperm binding to ZP were investigated.

In Experiment $\mathrm{VI}$, in addition to NP, other milk proteins such as SC and CG were used to modify TALP medium. Incubation of sperm and ZP with TALP containing milk proteins resulted in higher number of sperm bound to ZP compared with controls, except for CG1. SC and NP were more effective than CG in enhancing sperm binding to ZP.

NP is obtained from bovine milk through a process of microfiltration (Pierre et al. 1992). The structure of NP is a complex casein micelle with calcium phosphate incorporated in the micelle. SC is obtained by precipitation of NP and solubilization of the casein curd. During casein precipitation, the micellar structure is disrupted and the calcium phosphate is depleted. In our study, both the micellar (NP) and the nonmicellar (SC) forms of casein had a positive effect on sperm binding to ZP. These findings suggest that the mechanisms by which caseins affect sperm binding to ZP may be independent of the micelle structure. Batellier et al. (2000) demonstrated the importance of the micelle structure on preservation of stallion sperm motility during storage. Semen was diluted with media containing NP or SC and stored for 7 days at 4 or $15{ }^{\circ} \mathrm{C}$. At $15{ }^{\circ} \mathrm{C}$ storage, motility was higher when semen was diluted in media containing NP compared with SC; however, at $4{ }^{\circ} \mathrm{C}$, motility was similar when sperm were stored in media containing NP or SC. Apparently, micelles were partially destroyed at $4{ }^{\circ} \mathrm{C}$ (Rollema 1992), which would explain the lack of protective effect of NP at this temperature. Recently, Bergeron et al. (2007) demonstrated that these casein micelles present in skim milk are responsible for sperm protection during storage of bull semen. The proposed mechanism for sperm protection was that casein

Table 5 Effect of milk extenders and glucose on sperm binding to ZP.

\begin{tabular}{lcc}
\hline Treatment & ZP $(n)$ & Mean \pm s.E.M. \\
\hline TALP & 40 & $53 \pm 11^{*}$ \\
TGLU & 40 & $55 \pm 9^{*}$ \\
THGLU & 39 & $82 \pm 13^{*}$ \\
INRA & 40 & $151 \pm 10^{+}$ \\
EZM & 40 & $160 \pm 9^{+}$ \\
TSM & 38 & $167 \pm 14^{+}$ \\
\hline
\end{tabular}

${ }^{*}{ }^{\dagger}$ Values with different superscripts $\operatorname{differ}(P<0.05)$. Mean \pm S.E.M. numbers of sperm bound per bovine ZP in Experiment $\mathrm{V}$. 
Table 6 Effect of milk proteins on sperm binding to ZP.

\begin{tabular}{lccc}
\hline Treatment & $\begin{array}{c}\text { Milk protein }^{\mathrm{a}} \\
(\mathrm{mg} / \mathrm{ml})\end{array}$ & ZP $(n)$ & Mean \pm s.E.M. \\
\hline TALP & 0 & 60 & $27 \pm 4$ \\
CG1 & 1 & 60 & $41 \pm 9^{*, \pm}$ \\
CG3 & 3 & 60 & $61 \pm 8^{11, \mathrm{~A}}$ \\
SC1 & 1 & 60 & $78 \pm 9^{+}$ \\
SC3 & 3 & 60 & $102 \pm 16^{\mathbf{1}}$ \\
NP1 & 1 & 60 & $83 \pm 9^{\S}$ \\
NP3 & 3 & 60 & $97 \pm 21^{\mathrm{B}}$ \\
\hline
\end{tabular}

$*,+, \neq, \S, \|, \boldsymbol{\Phi}, \mathrm{A}, \mathrm{B}$ The superscripted preplanned comparisons differed as follow: ${ }^{*}{ }^{\dagger}(P<0.05) ;{ }^{\ddagger, \S}(P<0.01) ; "{ }^{\|}{ }^{\top}(P<0.01) ;{ }^{A}, \mathrm{~B}(P<0.01)$. Mean \pm S.E.M. numbers of sperm bound per bovine ZP in Experiment VI. a Milk proteins: CG, caseinoglycopeptide; SC, sodium caseinate; NP, native phosphocaseinate.

micelles sequester seminal plasma proteins that are known to stimulate continuous lipid removal from sperm plasma membrane. Because seminal plasma was largely removed by centrifugation in our study, it is unlikely that the mechanism by which milk proteins enhanced sperm binding to ZP would involve a direct interaction with seminal plasma proteins. In addition, because SC had a similar effect to NP on gamete binding, we suggest that SC could potentially be used as a substitute for NP in semen extenders used to preserve stallion semen during cold storage.

The mechanisms by which milk proteins enhance sperm binding to ZP are still unclear. One possible mechanism would involve initiation of sperm capacitation by milk proteins. Pommer et al. (2002) demonstrated that sperm incubated for $3 \mathrm{~h}$ in skim milk-based extender vs TALP had a higher intracellular calcium concentration and a higher percentage of cells undergoing the acrosome reaction after treatment with calcium ionophore, A23187, indicating that sperm were undergoing capacitation during incubation in skim milk-based extender. In contrast, Batellier et al. (2000) observed no increase in intracellular calcium concentrations when sperm were incubated in medium containing NP for $24 \mathrm{~h}$. As calcium evaluation was performed only after $24 \mathrm{~h}$ of incubation, intracellular calcium concentration could have been elevated and returned to basal levels by $24 \mathrm{~h}$, as described by Nagai et al. (1994). In our experiment, sperm and ZP were incubated for $2 \mathrm{~h}$ in medium containing $\mathrm{NP}$, and an increase in intracellular calcium in sperm could have occurred. In Experiments III and IV, sperm was exposed to NP for $\sim 30 \mathrm{~min}$, before being washed and placed in TALP. Short exposure time of sperm to NP could have been insufficient to result in an increase in intracellular calcium in sperm; therefore, no increase in sperm binding to ZP was observed. Our results provide multiple lines of evidence indicating that caseins are the molecules responsible for the dramatic increase in sperm binding to the ZP. Casein sequesters calcium with several binding constants and this characteristic may be highly relevant to the mechanism of facilitating binding, since calcium is often involved with adhesion of molecules. The casein might donate calcium in some subtle way that enhances adhesion during gamete co-incubation. Moreover, caseins may also interact with the sperm glycocalyx directly in ways that might more readily expose the sperm/oocyte receptors, or increase these proteins' sensitivity to the oocyte. Whichever the case is, they might be easily stripped from the sperm by centrifugation through Percoll. Our laboratory is currently investigating the effects of milk proteins on equine sperm capacitation and intracellular $\mathrm{Ca}^{2+}$ concentration in an attempt to elucidate the mechanisms responsible for increased sperm-ZP binding.

In conclusion, treatment responses of stallion sperm binding were similar for both bovine and equine $\mathrm{ZP}$, demonstrating suitability of bovine ZP for a binding assay for stallion sperm. Using this heterologous ZP-binding assay, we demonstrated that presence of milk and milk proteins during gamete interactions resulted in a three- to seven-fold increase in number of sperm bound to ZP. Due to potential differences in ZP characteristics between species, the mechanisms by which milk proteins affect stallion sperm binding to bovine ZP may be different from equine ZP. Therefore, studies on the mechanisms by which milk proteins enhance sperm binding to ZP would require a homologous system. These findings could further contribute to the development of assisted reproduction techniques for the horse, such as GIFT and IVF, as well as to increase our understanding of interactions between sperm and ZP.

\section{Materials and Methods}

Unless otherwise stated, all chemicals were purchased from Sigma-Aldrich.

\section{Bovine ZP}

Ovaries were recovered from cows at a slaughterhouse and transported to the laboratory in $0.15 \mathrm{M} \mathrm{NaCl}$ at $25-30{ }^{\circ} \mathrm{C}$ within $\sim 5 \mathrm{~h}$ of collection. Oocytes were aspirated from follicles with a 20-gauge needle, isolated under a stereomicroscope, and placed in modified Tyrode's medium (TALP; Table 7). All oocytes, with or without attached cumulus cells, were used. Oocytes were vortexed at maximum speed for 1$2 \mathrm{~min}$ to remove cumulus cells. Denuded oocytes were washed in TALP and stored in hyperosmotic salt solution $\left(1.5 \mathrm{M} \mathrm{MgCl}_{2}\right.$, $40 \mathrm{mM}$ HEPES, and $0.1 \%$ (w/v) PVP; Yanagimachi et al. 1979) at $5{ }^{\circ} \mathrm{C}$ for up to 3 months.

\section{Equine ZP}

Ovaries were recovered from mares at an abattoir and transported in saline to the laboratory within $\sim 4 \mathrm{~h}$ of collection. The antrum of follicles was scraped with a bone curette into HEPES-buffered TCM-199 with Hank's salts (Gibco Life Technologies, Inc.) into a Petri dish. Cumulus-oocyte 
Table 7 Compositions of media used ( $\mathrm{mM})$.

\begin{tabular}{|c|c|c|c|c|c|c|}
\hline Ingredient & TALP & TSM & TGLU & THGLU & EZM & INRA $^{a}$ \\
\hline Sanalac $(g / l)$ & - & 2.4 & - & - & 24 & - \\
\hline $\mathrm{NaHCO}_{3}$ & 35 & 35 & 35 & 35 & 18 & 4 \\
\hline HEPES & 10 & 10 & 10 & 10 & - & 20 \\
\hline Glucose & 5.5 & 5.5 & 89.5 & 163.5 & 272 & 67 \\
\hline Fructose & 84 & 84 & - & - & - & - \\
\hline Lactose & - & - & - & - & - & 126 \\
\hline $\mathrm{NaCl}$ & 37 & 37 & 37 & _- & - & 21.5 \\
\hline $\mathrm{KCl}$ & 10 & 10 & 10 & 10 & - & 5.4 \\
\hline $\mathrm{KH}_{2} \mathrm{PO}_{4}$ & 1 & 1 & 1 & 1 & - & 0.5 \\
\hline $\mathrm{MgSO}_{4}$ & 2.4 & 2.4 & 2.4 & 2.4 & - & 1 \\
\hline $\mathrm{CaCl}_{2}$ & 1.7 & 1.7 & 1.7 & 1.7 & - & 1.2 \\
\hline Na pyruvate & 0.18 & 0.18 & 0.18 & 0.18 & - & - \\
\hline Na lactate & 25.8 & 25.8 & 25.8 & 25.8 & - & - \\
\hline $\mathrm{BSA}(\mathrm{g} / \mathrm{l})$ & 3 & 3 & 3 & 3 & - & - \\
\hline $\mathrm{Na}_{2} \mathrm{HPO}_{4}$ & - & - & - & - & - & 0.8 \\
\hline $\begin{array}{l}\text { Native phosphoca- } \\
\text { seinate }(\mathrm{g} / \mathrm{l})\end{array}$ & - & - & - & - & - & 27 \\
\hline
\end{tabular}

For TALP, TSM, TGLU, and THGLU, pH was adjusted between 7.3 and 7.4.

aINRA 96 (Batellier et al. 1998).

complexes were identified using a stereomicroscope and placed in culture medium (TCM-199 with Earle's salts (Gibco), $5 \mu \mathrm{U} / \mathrm{ml}$ FSH (Sioux Biochemical, Inc., Sioux Center, IA, USA), 10\% (v/v) fetal bovine serum (FBS), and $25 \mu \mathrm{g} / \mathrm{ml}$ gentamicin sulfate). Oocytes were cultured for $24-26 \mathrm{~h}$ at $38.5{ }^{\circ} \mathrm{C}$ in an atmosphere of $5 \% \mathrm{CO}_{2}$ in air. After maturation, oocytes were denuded of cumulus cells by pipetting in a solution of $0.5 \%(\mathrm{w} / \mathrm{v})$ hyaluronidase in TCM-199 with $5 \%(\mathrm{v} / \mathrm{v})$ FBS. Only oocytes without a polar body were used for this experiment. Denuded oocytes were placed in hyperosmotic salt solution (Yanagimachi et al. 1979) and stored at $5{ }^{\circ} \mathrm{C}$ for up to 4 months.

\section{Stallions and sperm preparation}

Semen was obtained from six fertile stallions (A-F) individually housed at the Equine Reproduction Laboratory located at Colorado State University, Fort Collins. Stallions were maintained on a diet of mixed grass and alfalfa hay, vitamin and mineral supplement, and fresh water ad libitum according to the Institutional Animal Care and Use Committee protocols at the Colorado State University. For all experiments, each replicate was performed with sperm from a single stallion, and the semen was used to inseminate ZP within $\sim 60$ min from collection. After collection, semen was evaluated for motility and sperm concentration. Semen was diluted 1:3 with TALP and centrifuged at $400 \mathrm{~g}$ for $5 \mathrm{~min}$. Sperm were suspended with $1 \mathrm{ml}$ of the respective extender containing $35 \mu \mathrm{g} / \mathrm{ml}$ Hoechst 33342 (ICN Biomedical, Inc., Aurora, OH, USA), according to each experiment, and incubated for $15 \mathrm{~min}$ at $37^{\circ} \mathrm{C}$. After incubation, sperm were centrifuged at $400 \mathrm{~g}$ for $5 \mathrm{~min}$ and suspended at $2 \times 10^{6} \mathrm{sperm} / \mathrm{ml}$ in the respective extenders. Five microliters of sperm suspension were added to $45 \mu \mathrm{l}$ droplets of medium containing the $\mathrm{ZP}$, resulting in a final concentration of $2 \times 10^{5} \mathrm{sperm} / \mathrm{ml}$. Sperm motility for each treatment was determined subjectively immediately before and after co-incubation with the ZP.

\section{Binding assays}

Before each experiment, bovine or equine ZP was washed twice and incubated in TALP for at least $1 \mathrm{~h}$ at $38.5^{\circ} \mathrm{C}$ in an atmosphere of $5 \% \mathrm{CO}_{2}$ in air, except for Experiment IV-B. After incubation, ZPs ( $n=10$ per droplet) were randomly placed in droplets of $45 \mu \mathrm{l}$ medium (1 droplet per treatment) to which $5 \mu \mathrm{l}$ sperm suspension were added.

$\mathrm{ZP}$ and sperm were incubated for $2 \mathrm{~h}$ at $38.5^{\circ} \mathrm{C}$ in an atmosphere of $5 \% \mathrm{CO}_{2}$ in air. After incubation, ZPs were washed vigorously in four droplets of TALP using a small-bore, fire-polished, glass pipette to remove loosely bound sperm. ZPs were placed on a glass slide and covered with a coverslip supported by a mix of paraffin and petroleum jelly. ZPs were observed under an epifluorescence microscope (Eclipse E800, Nikon Instruments, Inc., Melville, NY, USA) equipped with a $360 / 40 \mathrm{~nm}$ band-pass excitation filter and a 460/50 nm bandpass emission filter. ZPs were observed under $400 \times$ magnification, and the number of sperm bound to ZP was recorded by a single technician, who was blinded as to the treatments.

\section{Experimental designs}

Experiment I: use of bovine ZP for ZP-binding assay with stallion sperm

Experiment I was designed to investigate the use of bovine ZP for a ZP-binding assay with stallion sperm. Bovine and equine ZPs were inseminated in parallel with semen diluted in TALP (Table 7) or in a skim milk-based extender (EZM; EZ-Mixin CST, Animal Reproduction Systems, Chino, CA, USA). Two ejaculates from each of the two stallions (A and D) were used. On each day of the experiment, semen from a single stallion was diluted with TALP, centrifuged, and suspended in TALP or EZM containing Hoechst 33342 as described earlier. After 15 min incubation at $37^{\circ} \mathrm{C}$, sperm were centrifuged and suspended in the same extender. Bovine and equine ZPs were inseminated with $5 \mu \mathrm{l}$ sperm suspended in TALP (BOV-TALP and EQ-TALP) 
or EZM (BOV-EZM and EQ-EZM) and incubated for $2 \mathrm{~h}$ at $38.5^{\circ} \mathrm{C}$ in an atmosphere of $5 \% \mathrm{CO}_{2}$ in air. After incubation, ZPs were washed and evaluated as described earlier to determine the number of sperm bound to ZP.

\section{Experiment II: effect of semen extenders on sperm binding to ZP}

Experiment II was designed to investigate the effects of semen extenders used for GIFT on binding of stallion sperm to bovine ZP. Two ejaculates from each of the three stallions (A, B, and C) were used on separate days. Semen was diluted with TALP, centrifuged, and suspended with one of the following extenders: lactose-EDTA (LAC; Cochran et al. 1984), TALP, EHS (ICP, Auckland, New Zealand), and EZM. Sperm were stained with Hoechst 33342 as described earlier, centrifuged, and suspended to $2 \times 10^{6} \mathrm{sperm} / \mathrm{ml}$ with the same extender. Sperm $(5 \mu \mathrm{l})$ were added to the droplets of TALP containing bovine $\mathrm{ZP}$, and the binding assay was performed as described earlier.

Experiment III: effect of centrifugation through Percoll on sperm binding to $Z P$

Experiment III was designed to investigate the effect of sperm centrifugation through a Percoll gradient on sperm binding to ZP. Two ejaculates from each of the two stallions ( $A$ and $B$ ) were used on separate days. After the initial centrifugation, sperm were suspended in $1 \mathrm{ml}$ TALP or EZM containing Hoechst 33342 and incubated for $15 \mathrm{~min}$ at $37^{\circ} \mathrm{C}$. After incubation, sperm diluted in TALP or EZM were washed by centrifugation at $400 \mathrm{~g}$ for $10 \mathrm{~min}$ (TALP and EZM) or through Percoll (TALP + P and $\mathrm{EZM}+\mathrm{P}$ ). Percoll gradients (90 and 45\% (v/v)) were prepared with TALP and $1 \mathrm{ml}$ of each gradient was used. Sperm were resuspended to $2 \times 10^{6} \mathrm{sperm} / \mathrm{ml}$ with TALP for groups TALP, TALP + $\mathrm{P}$, and EZM + P. In the EZM group, sperm were resuspended with EZM to the same concentration. Sperm $(5 \mu \mathrm{l})$ were added to the bovine $\mathrm{ZP}$, and the binding assay was performed as described earlier.

\section{Experiment IV: effect of EZM during sperm and ZP interaction}

Experiment IV was designed to investigate whether exposure of sperm or ZP to EZM before co-incubation would result in an increase in sperm binding to ZP. Two experiments (IV-A and IV-B) were performed. Two ejaculates from each of the two stallions were used for each experiment on separate days. In Experiment IV-A, after the initial centrifugation, sperm were diluted with TALP or EZM and stained with Hoechst 33342. Sperm diluted in TALP were centrifuged and resuspended in TALP. Sperm diluted in EZM were centrifuged and resuspended in TALP (SP-EZM) or EZM. Sperm preparations $(5 \mu \mathrm{l})$ were then added to the droplets containing bovine ZP, and the binding assay was performed as described earlier.

In Experiment IV-B, bovine ZPs were incubated in TALP alone (TALP and EZM) or TALP containing 10\% EZM (ZP-EZM) for $\sim 1 \mathrm{~h}$. Before the assay, all ZPs were washed twice in TALP and placed in $45 \mu \mathrm{l}$ TALP. Sperm were diluted with TALP or $E Z M$, stained, centrifuged, and suspended in the same extender. For groups TALP and ZP-EZM, ZPs were inseminated with sperm diluted with TALP. Sperm diluted with EZM were used for group EZM. Sperm $(5 \mu \mathrm{l})$ were added to the droplets containing $\mathrm{ZP}$, and the binding assay was performed as described earlier.

Experiment $V$ : effect of milk extenders and glucose on sperm binding to $Z P$

Experiment $V$ was designed to investigate the effects of milk extenders and glucose on sperm binding to ZP. TALP was modified (Table 7) with two concentrations of glucose (TGLU $=89.5 \mathrm{mM}$ and THGLU $=163.5 \mathrm{mM}$ ) and with the addition of skim milk (TSM $=2.4 \mathrm{mg} / \mathrm{ml}$ of Sanalac (HuntWesson, Inc., Fullerton, CA, USA)). The concentration of skim milk used in TSM corresponded to $10 \%$ of the concentration of skim milk found in EZM. In addition, INRA96 (IMV International Corp., Maple Grove, MN, USA) and EZM were used (Table 7).

Semen from one ejaculate from each of the four stallions (A-D) was centrifuged, suspended in TALP containing Hoechst 33342 , and incubated for $15 \mathrm{~min}$ at $37^{\circ} \mathrm{C}$. After centrifugation, sperm were suspended in one of the six extenders (TALP, TGLU, THGLU, TSM, INRA, and EZM). Bovine ZPs $(n=10 / \mathrm{tx})$ were placed in $45 \mu \mathrm{l}$ droplets of TALP, except the TSM group where ZPs were placed in a $45 \mu \mathrm{l}$ droplet of TSM. Sperm $(5 \mu \mathrm{l})$ from a single stallion were added to the $\mathrm{ZP}$, and the binding assay was performed as described earlier.

\section{Experiment VI: effect of milk proteins on sperm binding to ZP}

Experiment $\mathrm{VI}$ was designed to investigate the effects of SC, NP, and CG on sperm binding to ZP. NP was provided by Drs Phil Kelly and Brendan O'Kennedy (Teagasc, Dairy Products Research Centre, Moorepark, Ireland). SC and CG were purchased from Sigma Chemical Co. One ejaculate from each of the six stallions (A-F) was diluted with TALP, centrifuged, stained, and resuspended in TALP at $2 \times$ $10^{6} \mathrm{sperm} / \mathrm{ml}$. Bovine ZPs were washed and incubated in TALP for $1 \mathrm{~h}$ at $38.5^{\circ} \mathrm{C}$. After incubation, ZPs $(n=10 / \mathrm{tx})$ were placed in droplets of $45 \mu \mathrm{l}$ TALP containing no additions (TALP), 1 or $3 \mathrm{mg} / \mathrm{ml} \mathrm{SC}$ (SC1 and SC3), 1 or $3 \mathrm{mg} / \mathrm{ml} \mathrm{NP}$ (NP1 and NP3), and 1 or $3 \mathrm{mg} / \mathrm{ml}$ CG (CG1 and CG3). Sperm (5 $\mu \mathrm{l})$ were added to $\mathrm{ZP}$, and binding assays were performed as described previously.

\section{Statistical analysis}

Data were analyzed using the Statistical Analysis System software (SAS Institute, Inc., Cary, NC, USA). The droplet containing the $\mathrm{ZP}$ for each treatment was considered the experimental unit. The average number of sperm bound to ZP was calculated for each droplet and compared among groups. Data from each experiment were evaluated for homogeneity of variances and transformation was not necessary. Data were analyzed by the ANOVA with treatment and ejaculate in the model. For Experiments I through $\mathrm{V}$, pair-wise comparisons were separated by Tukey's HSD test. In Experiment VI, preplanned comparisons were made by least significant difference (LSD). 


\section{Declaration of interest}

The authors declare that there is no conflict of interest that could be perceived as prejudicing the impartiality of the research reported.

\section{Funding}

This work was supported by the benefactors of Preservation of Equine Genetics Program at Colorado State University, Fort Collins, CO.

\section{Acknowledgements}

The authors like to thank Drs Phil Kelly and Brendan O'Kennedy, Teagasc, Dairy Products Research Centre, Cork, Ireland, for generously providing the native phosphocaseinate and technical assistance. They also thank JoAnne E Stokes for technical assistance and Drs Katrin Hinrichs and Young-Ho Choi, College of Veterinary Medicine at Texas A\&M University, for providing equine oocytes.

\section{References}

Alvarenga MA \& Leao KM 2002 Hysteroscopic insemination of mares with low number of frozen thawed spermatozoa selected by Percoll gradient. Theriogenology 58 651-653. (doi:10.1016/S0093-691X(02)00897-X)

Arns MJ \& Shepherd RE 1994 Percoll gradient selection of equine spermatozoa enhances ability to bind and penetrate the zona pellucida. Theriogenology 41 158. (doi:10.1016/S0093-691X(05)80068-8)

Batellier F, Duchamp G, Vidament M, Arnaud G, Palmer E \& Magistrini M 1998 Delayed insemination is successful with a new extender for storing fresh equine semen at 15 degrees $C$ under aerobic conditions. Theriogenology 50 229-236. (doi:10.1016/S0093-691X(98)00130-7)

Batellier F, Gerard N, Courtens JL, Palmer E \& Magistrini M 2000 Preservation of stallion sperm quality by native phosphocaseinate: a direct or indirect effect? Journal of Reproduction and Fertility 56 69-77.

Braundmeier AG, Demers JM, Shanks RD \& Miller DJ 2004 The relationship of porcine sperm zona-binding ability to fertility. Journal of Animal Science 82 452-458.

Carnevale EM, Alvarenga MA, Squires EL \& Choi YH 1999 Use of noncycling mares as recipients for oocyte transfer and GIFT. Proceedings of the Annual Conference Society for Theriogenology p 44.

Carnevale EM, Maclellan LJ, Coutinho da Silva MA, Scott TJ \& Squires EL 2000 Comparison of culture and insemination techniques for oocyte transfer. Theriogenology 54 981-987. (doi:10.1016/S0093-691X(00) 00406-4)

Cochran JD, Amann RP, Froman DP \& Pickett BW 1984 Effects of centrifugation, glycerol level, cooling to $5^{\circ} \mathrm{C}$, freezing rate and thawing rate on the post-thaw motility of equine sperm. Theriogenology $\mathbf{2 2}$ 25-28. (doi:10.1016/0093-691X(84)90470-9)

Coutinho da Silva MA, Carnevale EM, Maclellan LJ, Seidel GE Jr \& Squires EL 2002 Effect of time of oocyte collection and site of insemination on oocyte transfer in mares. Journal of Animal Science $\mathbf{8 0}$ 1275-1279.

Coutinho da Silva MA, Carnevale EM, Maclellan LJ, Preis KA, Seidel GE Jr \& Squires EL 2004 Oocyte transfer in mares with intrauterine or intraoviductal insemination using fresh, cooled, and frozen stallion semen. Theriogenology 61 705-713. (doi:10.1016/S0093-691X(03) 00243-7)

Dean J 2004 Reassessing the molecular biology of sperm-egg recognition with mouse genetics. BioEssays 26 29-38. (doi:10.1002/bies.10412)

Del Campo MR, Donoso MX, Parrish JJ \& Ginther OJ 1990 In vitro fertilization of in vitro-matured equine oocytes. Journal of Equine Veterinary Science 10 18-22. (doi:10.1016/S0737-0806(06)80078-X)
Fazeli AR, Steenweg W, Bevers MM, deLoos FAM, vanden Broek J \& Colenbrander B 1993 Development of a sperm zona pellucida binding assay for bull sperm. Veterinary Record 132 14-16. (doi:10.1136/vr. 132.1.14)

Fazeli AR, Steenweg W, Bevers MM, vanden Broek J, Bracher V, Parlevliet J \& Colenbrander B 1995 Relation between stallion sperm binding to homologous hemizonae and fertility. Theriogenology 44 751-760. (doi:10.1016/0093-691X(95)00254-6)

Galli C, Crotti G, Turini P, Duchi R, Mari G, Zavaglia G, Duchamp G, Daels P \& Lazzari G 2002 Frozen-thawed embryos produced by ovum pick up of immature oocytes and ICSI are capable to establish pregnancies in the horse. Theriogenology 58 705-708. (doi:10.1016/ S0093-691X(02)00771-9)

Gamzu R, Yogev L, Amit A, Lessing J, Homonnai ZT \& Yavetz H 1994 The hemizona assay is of good prognostic value for the ability of sperm to fertilize oocytes in vitro. Fertility and Sterility 62 1056-1059.

Gamzu R, Yogev L, Paz G, Yavetz H \& Lichtenberg D 1997 Reduction of sperm cholesterol:phospholipid ratio is a possible mechanism for enhancement of human sperm binding to the zona pellucida following incubation with phosphatidylcholine liposomes. Biology of Reproduction 57 539-546. (doi:10.1095/biolreprod57.3.539)

Go KJ \& Wolf DP 1985 Albumin-mediated changes in sperm sterol content during capacitation. Biology of Reproduction 32 145-153. (doi:10.1095/ biolreprod32.1.145)

Green DPL 1997 Three-dimensional structure of the zona pellucida. Reviews of Reproduction 2 147-156. (doi:10.1530/ror.0.0020147)

He ZY, Brakebusch C, Fassler R, Kreidberg JA, Primakoff P \& Myles DG 2003 None of the integrins known to be present on the mouse egg or to be ADAM receptors are essential for sperm-egg binding and fusion. Developmental Biology 254 226-237. (doi:10.1016/S0012-1606 (02)00043-X)

Hoodbhoy T \& Dean J 2004 Insights into the molecular basis of sperm-egg recognition in mammals. Reproduction 127 417-422. (doi:10.1530/rep. 1.00181)

Hoshi K, Aita T, Yanagida K, Yoshimatsu N \& Sato A 1990 Variation in the cholesterol/phospholipid ratio in human spermatozoa and its relationship with capacitation. Human Reproduction 5 71-74.

Ivanova M \& Mollova M 1993 Zona-penetration in vitro test for evaluating boar sperm fertility. Theriogenology 40 397-410. (doi:10.1016/0093691X(93)90277-C)

Kenney RM, Bergman RV, Cooper WL \& Morse GW 1975 Minimal contamination techniques for breeding mares: techniques and preliminary findings. Proceedings of the 21st Annual Convention of the American Association of Equine Practitioners p 327.

Lazzari G, Crotti G, Turini P, Duchi R, Mari G, Zavaglia G, Barbacini S \& Galli C 2002 Equine embryos at the compacted morula and blastocyst stage can be obtained by intracytoplasmic sperm injection (ICSI) of in vitro matured oocytes with frozen-thawed spermatozoa from semen of different fertilities. Theriogenology 58 709-712. (doi:10.1016/S0093691X(02)00777-X)

Liu DY, Clarke GN, Lopata A, Johnston WIH \& Baker HWG 1989 A spermzona pellucida binding test and in vitro fertilization. Fertility and Sterility 52 281-287.

Meyers SA, Liu IKM, Overstreet JW, Vadas S \& Drobnis EZ 1996 Zona pellucida binding and zona-induced acrosome reactions in horse spermatozoa: comparisons between fertile and subfertile stallions. Theriogenology 46 1277-1288. (doi:10.1016/S0093-691X(96)00299-3)

Mugnier S, Dell'Aquila ME, Pelaez J, Douet C, Ambruosi B, De Santis T, Lacalandra GM, Lebos C, Sizaret PY, Delaleu B et al. 2009 New insights into the mechanisms of fertilization: comparison of the fertilization steps, composition, and structure of the zona pellucida between horses and pigs. Biology of Reproduction 81 856-870. (doi:10.1095/biolreprod. 109.077651)

Nagai T, Takenaka A, Mori T \& Hirayama M 1994 Effects of caffeine and casein phosphopeptides on fertilization in vitro of pig oocytes matured in culture. Molecular Reproduction and Development 37 452-456. (doi:10.1002/mrd.1080370412)

Nie GJ, Johnson KE \& Wenzel JG 2003 Pregnancy outcome in mares following insemination deep in the uterine horn with low numbers of sperm selected by glass wool/Sephadex filtration. Percoll separation or absolute number. Animal Reproduction Science 79 103-109. (doi:10. 1016/S0378-4320(03)00086-1) 
Oehninger S, Coddington CC, Scott R, Franken DR, Burkman LJ, Acosta AA \& Hodgen GD 1989 Hemizona assay: assessment of sperm dysfunction and prediction of in vitro fertilization outcome. Fertility and Sterility 51 665-670.

Osheroff JE, Visconti PE, Valenzuela JP, Travis AJ, Alvarez J \& Kopf GS 1999 Regulation of human sperm capacitation by a cholesterol effluxstimulated signal transduction pathway leading to protein kinase A-mediated up-regulation of protein tyrosine phosphorylation. Molecular Human Reproduction 5 1017-1026. (doi:10.1093/molehr/5.11. 1017)

Pantke P, Hyland JH, Galloway DB, Liu DY \& Baker HWG 1995 Development of a zona pellucida sperm binding assay for the assessment of stallion fertility. Biology of Reproduction Monograph Series $\mathbf{1}$ 681-687.

Pierre A, Fauquant J, Le Graet Y, Piot M \& Maubois JL 1992 Preparation de phosphocaseinate natif par microfiltration sur membrane. Lait 72 461-474. (doi:10.1051/lait:1992534)

Pommer AC, Linfor J \& Meyers SA 2002 Capacitation and acrosomal exocytosis are enhanced by incubation of stallion spermatozoa in a commercial semen extender. Theriogenology 57 1493-1501. (doi:10. 1016/S0093-691X(02)00659-3)

Rollema HS 1992 Casein association and micelles formation. In Advanced Dairy Chemistry-1: Proteins, pp. 111-140. Ed PF Fox. New York: Elsevier Science BV.

Sinowatz F, Wessa E, Neumuller C \& Palma G 2003 On the species specificity of sperm binding and sperm penetration of the zona pellucida. Reproduction in Domestic Animals 38 141-146. (doi:10.1046/j.14390531.2003.00401.x)

Visconti PE, Galantino-Homer H, Ning X, Moore GD, Valenzuela JP, Jorgez CJ, Alvarez JG \& Kopf GS 1999a Cholesterol efflux-mediated signal transduction in mammalian sperm. beta-cyclodextrins initiate transmembrane signaling leading to an increase in protein tyrosine phosphorylation and capacitation. Journal of Biological Chemistry 274 3235-3242. (doi:10.1074/jbc.274.5.3235)

Visconti PE, Stewart-Savage J, Blasco A, Battaglia L, Miranda P, Kopf GS \& Tezon JG $1999 \mathrm{~b}$ Roles of bicarbonate, CAMP, and protein tyrosine phosphorylation on capacitation and the spontaneous acrosome reaction of hamster sperm. Biology of Reproduction 61 76-84. (doi:10.1095/ biolreprod61.1.76)

Visconti PE, Ning X, Fornes MW, Alvarez JG, Stein P, Connors SA \& Kopf GS 1999 C Cholesterol efflux-mediated signal transduction in mammalian sperm: cholesterol release signals an increase in protein tyrosine phosphorylation during mouse sperm capacitation. Developmental Biology 214 429-443. (doi:10.1006/dbio.1999.9428)

Yanagimachi R 1994 Mammalian fertilization. In The Physiology of Reproduction, 5th edn, pp 189-317. Eds E Knobil \& JD Neill. New York: Raven Press.

Yanagimachi R, Lopata A, Odom CB, Bronson RA, Mahi CA \& Nicolson GL 1979 Retention of biologic characteristics of zona pellucida in highly concentrated salt solution: the use of salt-stored eggs for assessing the fertilizing capacity of spermatozoa. Fertility and Sterility 31 562-574.

Received 29 March 2011

First decision 9 May 2011

Revised manuscript received 8 March 2012

Accepted 12 March 2012 